\title{
Standardization Issues of Mobile Usability
}

\author{
https://doi.org/10.3991/ijim.v14i07.12129 \\ Radka Valerieva Nacheva \\ University of Economics - Varna, Varna, Bulgaria \\ r.nacheva@ue-varna.bg
}

\begin{abstract}
The benefits of maintaining standards can be summarized from different perspectives: consumers, businesses, governments, economically. They inform and shape the products we buy, ensuring quality and safety. Standardizing in dynamic activities such as the development of mobile applications and devices can be not easy task. In this regard, the aim of the current paper is to revise some international standard towards usability problems of mobile user interfaces and to analyze the possibility of standardization of mobile usability.
\end{abstract}

Keywords - Mobile usability, international standards, interface design, standardization problems

\section{Introduction}

The specifics of mobile application development are mainly related to the limitations imposed by the devices themselves. For example, low power consumption, small physical sizes, various input methods (so called "multimodality" ${ }^{1}$ ), limited memory, processing power and screen size, poor connectivity to mobile and internet networks. It is also necessary to take into account the fact that the use of mobile technologies takes place in different, often changing environments in which users are exposed to changes in their environments. For example, different noise levels, changes in brightness, temperature, etc. These affect the attention of individuals, which is a prerequisite for making more mistakes when working with mobile applications.

The ease of use, efficiency, and satisfaction of using mobile devices and the various types of software designed for them is related to the so-called "mobile usability". With the active use of mobile devices, there is a recent need for mobile applications to be designed in a user-friendly way. Efficiency affects fast and easy navigation in mobile applications.

Based on our previous research [1], we can define usability as a qualitative criterion that applies not only to the user interface of a product, but also to its functionality, considered in a specific context of use. When determining the usability of a product, account should be taken of the ease of its use, which, according to the author of this

1 It is associated with the provision of multiple approaches for human-technology interaction so that users are able to work with most of their senses. Most technologies provide a three-modal interaction approach - visual, auditory and tactile, with other senses usually excluded. 
study, it covers ease of learning, ease of remembering, tolerance for errors. If the additional factors that determine usability are to be defined and which are properly reflected in its evaluation by methods and software, then these are: efficiency, productivity, ease of interaction, security and utility, set out in a specific context of use and always considered in combination to achieve satisfaction for users using the technology concerned. This requires that the term "usability" be defined as a context-dependent criterion that does not have a single measurement.

In practice, there are standards and recommendations for creating usable applications, including methods and tools that are used to determine usability. For the most part, their wording is general, universal in nature, not concretely targeting the specifics of the particular type of mobile application.

In this regard, the aim of the current paper is to revise some international standard toward to usability problems of mobile user interfaces and to analyze the possibility of standardization of mobile usability.

\section{International Standards Comparison}

For successful communication between human and technology, the user interface must be developed in a usable way. This can be done by:

- Complying with basic design rules and principles and / or standards that are generally applicable when creating a human-machine interface.

- Complying with the rules / principles and / or standards related to development of mobile application interfaces.

- Applying mobile app user interface design templates that integrate good and commonly used design practices for this type of applications.

On the one hand, design rules, principles, standards and patterns are usually designed based on the experience of design professionals and scientists examining the individual characteristics of users and the specifics of the environment and their impact on people of the technologies concerned. They are the result of studies in the field of humancomputer interaction.

On the other hand, the specific characteristics of mobile devices, the current work environment and mobile and wireless networks pose a number of significant challenges in exploring the usability of mobile applications. In this regard, it should be noted that problems with the usability of the user interfaces, not only of the mobile but also of the software applications as a whole, arise from the failure of some of the best practices, defined in international standards, by leading specialists in the field and / or software companies developing the operating systems running the applications. They are usually defined as recommendations, rules, or principles. These are the result of years of research teams working on the testing of specific technology groups of users. The standards should be relevant to creating user-oriented technologies. The difficulty in the situation stems from the fact that there is no clear definition of the meaning of the term "usability". However, developing usability standards for different types of technology 
would bring a number of positives, such as independence, balance and authority, to promote consistency.

Some standards are defined by the International Standards Organization, but other international organizations are working in this direction too. Some of them consider the usability of mobile applications, while others look at high concept issues.

At the time of the research on the paper, we found that the usability of mobile technology user interfaces is concerned in the standards ISO/IEC 24755:2007, ISO/IEC 18021:2002 and ISO/IEC TR 15440:2016.

ISO/IEC 24755:2007 "Information technology — Screen icons and symbols for personal mobile communication devices" defines a consistent set of screen icons and symbols intended for mobile communication devices (for example, mobile phones and personal digital assistants) [4]. This international standard provides a set of icons for the use of personal information of applications related to device management. Although each platform has specific requirements for the applications it develops, including in terms of user interface, and full design guides are provided, maintaining such a standard is useful as it aims to provide a universal approach to creating icons. This also helps to ensure unity in the look of the same application created for different platforms.

The standard ISO/IEC 18021:2002 "Information technology - User interfaces for mobile tools for management of database communications in a client-server model" defines only features of the user interface for managing communication when exchanging data between a mobile device - client and server. Two user interfaces are defined for:

- Approving when update a client database or when data in a client database are transmitted to another database.

- Providing feedback to the user after the client or database data of the server running the mobile device has been updated.

ISO/IEC TR 15440:2016 "Information technology — Future keyboards and other input devices and entry methods" covers the following aspects [6]:

- Different login requirements, consistent with national and international practices and support for cultural and linguistic diversity

- Recognition of requirements in accordance with the comfort of use (regardless of the target group - children, adults, people with disabilities)

- Improving user productivity when entering data

- Enhancements to keyboards and related input devices and methods required for newtype applications (such as virtual reality ones)

- Input requirements for virtual keyboards

- Labeling (permanent and temporary labels), functional symbols and icons.

Another standard-setting organization that sets international standards but focuses only on the Web is the World Wide Web Consortium (W3C). It defines standards for mobile web applications in the form of the Mobile Web Initiative, which addresses APIs related to graphical and multimedia elements, forms, user interactions with devices, usability and accessibility, data storage, customization of applications across 
devices, sensor and hardware integration, personal information management, network settings, payments, security issues, and more. In addition, sample architectures and developer guidelines are provided along with sample code to help developing mobile web applications. The advantage of maintaining such a standard is that various aspects of the lifecycle of this type of application are fully addressed, in terms of applying technology and good practices. Detailed instructions are given for developers when working with them. But on the other hand, not all types of mobile applications (intended for a specific platform - native, and hybrid / cross-platform - or hybrid / cross-platform) are covered, and there are no stated principles for the design of usable mobile applications. Mobile app user interface design templates or other examples of usable composition of interface elements are also not provided.

European Telecommunications Standards Institute (ETSI) creates globally applicable standards for ICT-enabled systems, applications and services. It defines many standards related to human factors and mobile communication. Aspects of mobile usability are concerned in some of the standards. They refer to related ISO and W3C standards. ETSI EG 202048 Human Factors (HF); Guidelines on the multimodality of icons, symbols and pictograms gives "guidelines for the design and use of multimodal symbols using a Design for All approach” [12]. ETSI EG 202416 Human Factors (HF); User Interfaces; Setup procedure design guidelines for mobile terminals and services "provides user interface design guidelines for setup procedures, applicable to mobile terminals and services throughout the product life-cycle" [13]. ETSI TR 102972 Human Factors (HF); User Interfaces; Generic user interface elements for 3G/UMTS mobile devices, services and applications "addresses the user interfaces of 3G/UMTS-enabled devices, services and applications from the end users' perspective, and provides generic design, development, deployment and evaluation recommendations" [15]. ETSI EG 202132 Human Factors (HF); User Interfaces; Guidelines for generic user interface elements for mobile terminals and services "addresses key issues from the end user's perspective, providing guidance on proposed generic user interface elements for basic and advanced mobile terminals, services and certain aspects of application handling" [16]. Its aim is providing simplified access advanced functions of mobile communication. ETSI EG 202191 Human Factors (HF); Multimodal interaction, communication and navigation guidelines "identifies key issues, solutions and actions for multimodal interaction, communication and navigation at the user interface with ICT systems and terminals. It specifically addresses the usage context of transactional interactions for independent living" [17].

It should be noted that the presented standards partially mention the usability of mobile applications but do not make comprehensive recommendations, which can be considered a disadvantage. Not all the features of mobile devices and their applications are taken into account. One of the main reasons for this is that such type of technology is evolving too dynamically, and creating an exhaustive standard that includes definitions, usability criteria, recommendations for creating usable user interfaces, testing methods and evaluation, it takes a time - in the order of 5 years.

In such a situation, it is correct to look for standards that address the usability problems of software applications at a higher, conceptual level. The only such and comprehensive standard to date that "provides a detailed guide to user interface design" [2] is 
ISO 9241. It covers many aspects for people working with a computer. The part of the standard that sets universal guidelines for developing usable software user interfaces is Part 110: Dialogue Principles, and Part 129: Guidance on software individualization can be used as a supplementary part. Part 210 deals with the so-called "User-Centered Design" (UCD) or "Human-Centered Design" (HCD) which were part of ISO 13407: 1999. Based on the UCD definition as "an interactive systems development approach that focuses on creating usable systems" [7], it can be defined as a multidisciplinary activity that incorporates ergonomics, performance and productivity enhancing techniques working conditions of people with a system and neutralizing the possible adverse effects of its use on human health, safety and productivity. The principles behind the custom design described in Part 210 of ISO 9241 and adopted by those skilled in the art are as follows [3]:

- Active involvement of users of the system and a clear understanding of the tasks they are to perform

- Proper distribution of functions between users and technology

- Reuse of design solutions

- Multidisciplinary design.

In addition, UCD activities defined in the standard build the guideline for creating user-oriented designs:

- Learning and specifying the context of use: this involves knowing the user, the environment of use, and the tasks for which he / she uses the product

- Specifying user and organizational requirements: This activity refers to defining product success criteria for user tasks, such as how quickly a typical user must be able to complete a product task. This includes setting design guidelines and imposing various restrictions

- Specifying user and organizational requirements: such activity refers to defining product success criteria for user tasks, such as how quickly a typical user must be able to complete a product task. This includes setting design guidelines and imposing various restrictions

- Design evaluation in accordance with the imposed requirements: the usability of the designs is evaluated according to user tasks.

It should be noted that neither the principles nor the activities in Part 210 of ISO 9241 set specific rules that are advisable to follow when creating user-oriented designs, but are essential in defining the major factors that influence the creation of usable user interfaces. It can be concluded that the restrictive conditions that are taken into account relate to the audience, the context of use and the system requirements defined, both by users and by the organization.

The series and other parts of ISO 9241 focus, in general, on the basics of ergonomics and the basics of human-computer interaction, for both software and hardware; accessibility of the software; user-oriented design of interactive systems, etc., which are not relevant to the present study. 
Due to the limitations imposed by the format of the study submission, it is not possible to look at all the rules defined, but it can be summarized that Part 110 sets specific criteria for evaluating the dialogue that would serve as guidance for the desired end result of the user interface design process, namely its usability. The criteria are as follows: acceptability of the dialogue when performing tasks, informativeness, compliance with the needs of the target audience, possibility for self-education, controllability, resistance to errors, adaptability to the individual characteristics of users.

The standard ETSI EG 202116 Human Factors (HF); Guidelines for ICT products and services; "Design for All" is based on ISO 13407: 1999 too. It is "applicable to ICT products with a user interface that are connectable to all kinds of fixed and mobile telecommunications networks" [14]. As it is mentioned in the document designing ICT products and services results in a three-level model:

1. Mainstream products designed according to good Human Factors practice, incorporating considerations for people with impairments, that can be used by a broad range of users

2. Products that are adaptable to permit the connection of assistive technology devices 3. Specially designed or tailored products for very disabled users.

In Table 1, we synthesize some of the characteristics of the international standards presented so far, including the period of revision.

Table 1. Comparison of International Standards

\begin{tabular}{|l|c|c|l|}
\hline \multicolumn{1}{|c|}{ Standard } & Mobile $^{\mathbf{2}}$ & Revision $^{\mathbf{3}}$ & \multicolumn{1}{c|}{ Period $^{\mid}$} \\
\hline ISO/IEC 24755:2007 & yes & 2017 & 5 years \\
\hline ISO/IEC 18021:2002 & yes & 2017 & 5 years \\
\hline ISO/IEC TR 15440:2016 & yes & 2016 & 5 years \\
\hline ISO 13407:1999 & no & 1999 & 5 years \\
\hline ISO 9241-210:2019 & no & 2019 & 5 years \\
\hline ISO 9241-161:2016 & no & 2016 & 5 years \\
\hline ISO 9241-129:2010 & no & 2016 & 5 years \\
\hline ISO 9241-110:2006 & no & 2018 & 5 years \\
\hline Standards for Web Applications on Mobile & yes & 2015 & $1-2$ years \\
\hline ETSI EG 202 116 & yes & 2009 & - \\
\hline ETSI EG 202 048 & yes & 2002 & - \\
\hline ETSI EG 202 416 & yes & 2006 & - \\
\hline ETSI TR 102 972 & yes & 2009 & - \\
\hline ETSI EG 202 132 & yes & 2004 & - \\
\hline ETSI EG 202 191 & yes & 2003 & - \\
\hline
\end{tabular}

Of the considered standards, only ISO/IEC 24755:2007, ISO/IEC 18021:2002 and ISO/IEC TR 15440:2016 affect in some respects the usability of the user interface of mobile applications and devices, but do not make exhaustive recommendations, which

\footnotetext{
2 Relation to mobile user interfaces design or usability.

3 The year in which the latest version of the standard was officially approved and published
} 
is a disadvantage. Not all features of mobile devices and their applications are taken into account.

The W3C standards fully address various aspects of the lifecycle of web application, in terms of applying technology and good practices. Detailed instructions for developers are provided. On the other hand, not all types of mobile applications (intended for a specific platform - native, and hybrid or still multi-platform - hybrid / cross-platform) are covered. There are no design principles for usable mobile applications. There are also no user interface design templates or other examples of usable composition of interface elements.

The ISO 9241 and ISO 13407: 1999 standards address the problems of user-oriented interfaces, in particular usability, but do not affect the specifics of mobile applications. ISO 13407: 1999 was discontinued and, at the time of the paper studies, was revised by ISO 9241-210: 2010, which is currently ISO 9241-210: 2019.

ISO 9241 covers many aspects of the ergonomics of human-computer interaction, including and the principles of dialogue (Part 110) and user-oriented designs of interactive systems (Part 210). Parts 129 and 161 may be used as supplements to set up dialogue principles and a guide for creating user interface elements, but without taking into account the particular context of use.

Part 210 of the ISO 9241 standard sets out the principles and activities of user-centered designs that are perceived by those skilled in the art as fundamental. Implementing principles and activities defined in the standards in the context of mobile applications should provide a good basis for building usable interfaces.

ETSI standards mostly affect mobile usability, but they have not been updated in recent years. The most recent of these standards is from 2010. Unfortunately, the renewal period has not been described, only the establishment procedure [18]. We consider this a disadvantage in view of the rapid development of technology and changes in design trends. As an advantage, we can say that they are available online for free. The standards provide useful and detailed guidance related to developing the interface of mobile devices.

It should be noted that neither the principles nor the activities in all the standards set specific rules that are advisable to follow when creating user-oriented designs, but are essential in defining the main factors that influence usable user interface design. It can be concluded that the restrictive conditions that are taken into account relate to the audience, the context of use and the defined requirements for the system, both by users and by companies.

\section{Conclusion}

In summary, as noted by some authors [11], adherence to a specific standard may create an unnecessary restriction on the product's innovation, in particular its design. However, even if not sufficiently detailed, the standard "describes principles for user interface design, not working solutions." It should also be borne in mind that due to the rapid development of information and communication technologies, the standard "may 
quickly become out of date" [11]. All international standards shall be reviewed at least every five years, as reflected in Table 1.

Maintaining standards in dynamic areas, such as the development of mobile technologies, is not always possible but also effective. Therefore, it is necessary to pay attention to design recommendations and principles provided by professionals working in the field of mobile technologies and companies developing mobile operating systems.

\section{References}

[1] Nacheva, R. (2017). Architecture of Web-Based System for Usability Evaluation of Mobile Applications", "Izvestiya" - Journal of University of Economics - Varna, 61(2): 187 - 201.

[2] ISO/DIS 9241-11.2:2018 Ergonomics of human-system interaction - Part 11: Usability: Definitions and concepts.

[3] ISO 9241-210:2019 Ergonomics of human-system interaction - Part 210: Human-centred design for interactive systems. https://doi.org/10.3403/30388991

[4] ISO/IEC 24755:2007 Information technology — Screen icons and symbols for personal mobile communication devices.

[5] ISO/IEC 18021:2002 Information technology — User interfaces for mobile tools for management of database communications in a client-server model.

[6] ISO/IEC TR 15440:2016 Information technology — Future keyboards and other input devices and entry methods.

[7] ISO 13407:1999 Human-Centred Design Processes for Interactive Systems, Geneva, Switzerland: ISO.

[8] ISO 9241-161:2016 Ergonomics of human-system interaction - Part 161: Guidance on visual user-interface elements.

[9] ISO 9241-129:2010 Ergonomics of human-system interaction - Part 129: Guidance on software individualization.

[10] ISO 9241-110:2006 Ergonomics of human-system interaction — Part 110: Dialogue principles.

[11] Bevan, N. (2006). International Standards for HCI and Usability. International Journal of Human Computer Studies, 55(4): 533 - 552. https://doi.org/10.1006/ijhc.2001.0483

[12] ETSI EG 202048 V1.1.1 (2002-08) ETSI Guide Human Factors (HF); Guidelines on the multimodality of icons, symbols and pictograms, 2002.

[13] ETSI EG 202416 Human Factors (HF); User Interfaces; Setup procedure design guidelines for mobile terminals and services, 2006.

[14] ETSI EG 202116 Human Factors (HF); Guidelines for ICT products and services; "Design for All", 2009.

[15] ETSI TR 102972 Human Factors (HF); User Interfaces; Generic user interface elements for 3G/UMTS mobile devices, services and applications, 2009.

[16] ETSI EG 202132 Human Factors (HF); User Interfaces; Guidelines for generic user interface elements for mobile terminals and services, 2004.

[17] ETSI EG 202191 Human Factors (HF); Multimodal interaction, communication and navigation guidelines, 2003.

[18] ETSI. Standards making. [Online]. Available: https://www.etsi.org/standards/standardsmaking. [Accessed October 27, 2019]. 


\section{Author}

Radka Nacheva is a chief assistant professor who work at Department of Informatics, Faculty of Informatics, University of Economics - Varna. She holds a PhD in Informatics from the same University. Her research interests are in Human-Computer Interaction, Cognitive Science and Software Accessibility. Dr. Nacheva teaches in Web technologies, Software Design and Operating Systems. She is currently a head of a project "Contemporary Approaches to the Integration of Mobile Technologies in Higher Education".

Article submitted 2019-10-30. Resubmitted 2019-12-07. Final acceptance 2019-12-10. Final version published as submitted by the authors. 


iJIM - Vol. 14, No 7, 2020

\section{Imprint}

iJIM - International Journal of Interactive Mobile Technologies

http://www.i-jim.org

Editor-in-Chief

Michael E. Auer, IAOE, Vienna, Austria

\section{Associate Editors-in-Chief}

Arthur Walter Edwards, Universidad de Colima, Mexico

Thrasyvoulos Tsiatsos, Aristotle University of Thessaloniki, Greece

\section{Section Editor}

Apostolos Gkamas, Greece

\section{Regional Associate Editors}

Abdallah Yusuf Al-Zoubi, Associated Editor Middle East Rhena Delport, Associated Editor Africa

Daphne Economou, Associated Editor Western Europe Arthur Walter Edwards, Associated Editor Latin America Ferial Khaddage, Associated Editor Australia Tzu-Chien Liu, Associated Editor Asia

Doru Ursutiu, Associated Editor Eastern Europe

Mudasser Fraz Wyne, Associated Editor North America

\section{Technical Director}

Sebastian Schreiter, Lagorce, France

\section{Editorial Board}

A. Y. Al-Zoubi, Princess Sumaya University for Technology Amman, Jordan Yacob Astatke, Morgan State University, United States

Rhena Delport, University of Pretoria, South Africa

Daphne Economou, Senior Lecturer, University of Westminster, United Kingdom Arthur Walter Edwards, Universidad de Colima, Mexico, Mexico Juan Antonio Guerrero-lbáñez, University of Colima, Mexico Hyo-Joo Han, Georgia Southern University Markus Feisst, University of Applied Sciences Offenburg, Germany Ferial Khaddage, Lecturer in Mobile Computing and I.T., Australia Dr. Kinshuk, Athabasca University Canada Adamantios Koumpis, ALTEC Software S.A., Greece Tzu-Chien Liu, National Central University, Taiwan Hiroaki Ogata, Tokushima University, Japan

Andreas Pester, Carinthia University of Applied Sciences, Austria Raul Aquino Santos, University of Colima, Mexico

Doru Ursutiu, University Transilvania of Brasov, Romania Mudasser Fraz Wyne, National University, USA

\section{Indexing}

International Journal of Interactive Mobile Technologies is indexed in Elsevier Scopus, INSPEC, Ulrich, DOAJ, EBSCO, Google Scholar, and DBLP.

\section{Publication Frequency}

Bimonthly

\section{Publisher}

International Association of Online Engineering (IAOE)

Kirchengasse 10/200

A-1070 WIEN

Austria 